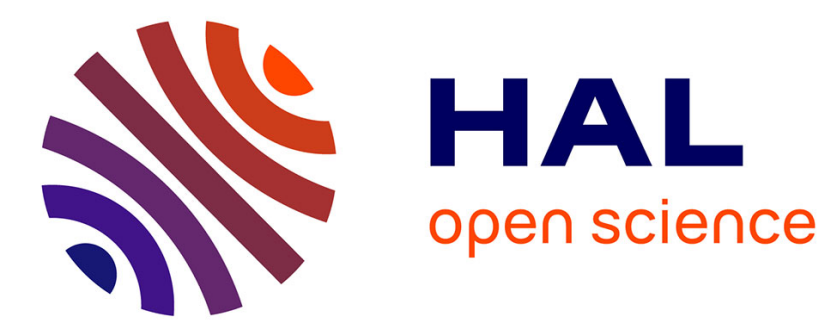

\title{
Multi-Reference Shape Priors for Active Contours
}

Alban Foulonneau, Pierre Charbonnier, Fabrice Heitz

\section{To cite this version:}

Alban Foulonneau, Pierre Charbonnier, Fabrice Heitz. Multi-Reference Shape Priors for Active Contours. International Journal of Computer Vision, 2009, 81, pp.68-81. 10.1007/s11263-008-0163-3 . hal-00873962

\section{HAL Id: hal-00873962 \\ https://hal.science/hal-00873962}

Submitted on 16 Oct 2013

HAL is a multi-disciplinary open access archive for the deposit and dissemination of scientific research documents, whether they are published or not. The documents may come from teaching and research institutions in France or abroad, or from public or private research centers.
L'archive ouverte pluridisciplinaire HAL, est destinée au dépôt et à la diffusion de documents scientifiques de niveau recherche, publiés ou non, émanant des établissements d'enseignement et de recherche français ou étrangers, des laboratoires publics ou privés. 


\title{
Multi-Reference Shape Priors for Active Contours
}

\author{
Alban Foulonneau ${ }^{1}$, Pierre Charbonnier ${ }^{1}$, and Fabrice Heitz ${ }^{2}$ \\ 1 ERA 27 LCPC, Laboratoire des Ponts et Chaussées 11 rue Jean Mentelin, B.P. 9, 67035 Strasbourg, \\ France \\ Alban.Foulonneau@uha.fr, Pierre.Charbonnier@equipement.gouv.fr \\ 2 Laboratoire des Sciences de l'Image, de l'Informatique et de la Télédétection, UMR 7005 CNRS, \\ Strasbourg I University, Bd Sébastien Brandt, 67400 Illkirch, France
}

heitz@lsiit.u-strasbg.fr

\begin{abstract}
In this paper, we present a new way of constraining the evolution of an active contour with respect to a set of fixed reference shapes. This approach is based on a description of shapes by the Legendre moments computed from their characteristic function. This provides a region-based representation that can handle arbitrary shape topologies. Moreover, exploiting the properties of moments, it is possible to include intrinsic affine invariance in the descriptor, which solves the issue of shape alignment without increasing the number of d.o.f. of the initial problem and allows introducing geometric shape variabilities. Our new shape prior is based on a distance, in terms of descriptors, between the evolving curve and the reference shapes. Minimizing the corresponding shape energy leads to a geometric flow that does not rely on any particular representation of the contour and can be implemented with any contour evolution algorithm. We introduce our prior into a two-class segmentation functional, showing its benefits on segmentation results in presence of severe occlusions and clutter. Examples illustrate the ability of the model to deal with large affine deformation and to take into account a set of reference shapes of different topologies.
\end{abstract}

\section{ECCV SPECIAL ISSUE}




\section{Introduction}

Exploiting high-level information about expected objects to ease the interpretation of low-level cues extracted from images, following the mechanism of visual attention, may be highly beneficial in applications such as image segmentation. In this spirit, incorporating global shape constraints into active contours traces back to pioneering works such as [1-4]. It has recently received an increasing attention in the context of implicit representations (see e.g. [5-9] and references therein). In the present paper, we present a novel approach for constraining the geometry of an evolving active contour toward a set of reference shapes.

\subsection{Relationship to prior work}

Our approach bears similarities with several previously published contributions. There are several ways of enforcing shape constraints on active contours. While alternatives have been proposed in $[6$, 9-11], in the most usual approach, an additional prior term is incorporated into the segmentation energy functional $[2,7,12-16]$. This framework includes a trade-off parameter which allows tuning the balance between the data fidelity term and the shape prior according to the level of noise and the confidence in the model.

Most of the time, the shape prior is based on a similarity measure between the evolving shape and a reference one, which may be either a given silhouette $[8,17]$ or the result of a (pre)segmentation stage $[15,18]$ or the outcome of some learning procedure $[2,5,7,9,13,14]$.

While several authors employ a parametric representation of curves $[2,4,5]$, or geometric differential representations [19], a vast majority of recent papers consider non-parametric models. In particular, signed distance functions have become popular $[6,7,9,13,14,16]$. An interesting feature of such implicit representations is that they do not constrain shape topology. However, they are most of the time linked to a particular implementation, namely the level sets framework while in certain contexts, faster implementations that also handle changes of topology (e.g. [20]) may be preferable.

The first important issue that must be dealt with when using a reference shape is the question of variability. Variations of the shape away from a reference template are, in the majority of existing works, handled using statistical models, even if a framework that accounts for geometric transformations of the reference shape was proposed in [8]. Many models are based on standard Principal Component Analysis (PCA) [3, 4,6,9,14] which involves Gaussian distributions. To better model real-world shape distributions, which may be arbitrarily complex, Gaussian kernel space density estimation [5] and, more recently, Parzen kernel density estimation [16] were proposed.

A second issue is the question of shape alignment. Pose parameters (rotation, translation and scaling) are generally taken into account in an explicit fashion [6-9,13,14], which increases the number of d.o.f. of the problem, and leads to systems of coupled partial differential equations (PDE's). To overcome these problems, intrinsic alignment was proposed: in $[4,12]$ for explicit snakes implementations, in $[16,17]$ for implicit representations in the case of translation and scale invariance, and extended in $[21]$ to the affine case.

\subsection{Contributions of our work}

The approach reported in the present paper combines a compact, parametric representation of shapes (introduced by the authors in $[17,21]$ for the modeling of single shapes) with curve evolution theory. More specifically, this parametric description is based on Legendre moments computed from the 
characteristic function of a shape. Such region-based representation shares with implicit representations $[6,7,13,14]$ the appealing property of dealing with arbitrary shape topologies. The advantage of our model over those based on signed distance function is that it is not bound to any particular implementation.

Moreover, as we have shown in [21], the geometric information about shape, carried by moments, can be exploited to make our shape descriptor intrinsically affine-invariant. This avoids the problem of pose estimation and introduces supplementary, geometrical variability in the model. In contrast with methods that explicitly take into account pose parameters $[6-9,13,14]$, no additional estimation is needed. Note that, among the intrinsically invariant models previously proposed, the representations in $[4,12]$ cannot handle complex topologies, in contrast with the approaches in $[16,17]$, but the latter are limited to translation and scale invariance. To our knowledge, only the method proposed in [8] has further degree of invariance: it can handle projective transformations but using an explicit formulation, however. Finally, note that the pose parameters are readily available as a by-product of our method, which can be crucial in certain applications.

The model presented in [21] was limited to the single-reference, affine-invariant case. In the present paper, we address the multi-reference case, i.e. multiple reference shapes are simultaneously considered, in the spirit of [16]. More precisely, the proposed shape prior is defined based on a distance, in terms of descriptors, between the evolving curve and the reference shapes. This is obviously a way of introducing variability into the model. As illustrated by experimental results, further variations may be dealt with by combining the multi-reference model with affine invariance. To our knowledge, such results are completely novel in the domain. Introducing strong geometric invariance, especially in the multi-reference case, obviously yields intricate energy landscapes with many local minima, making the optimization more difficult. We also show that some geometric transformations such as rotations, may be handled using the multi-reference model in conjunction with a lower level of geometric invariance. In the multi-reference context, the distance between descriptors constitutes an interesting feature for shape recognition or pose estimation.

Finally, a unique evolution equation for the active contour is derived using the formalism of shape derivative and classical differentiation rules as proposed in [22] by Aubert et al. Thanks to the ability of the model to change topology during evolution, automatic initialization of the active contour is also possible, whatever the topology of the final target shape. Moreover, as already stated, neither the model nor the evolution equation depend on any implementation consideration. Consequently, both level sets [23] or spline-snakes [20] implementations may, for instance, be considered.

The remainder of the paper is structured as follows. In Sec. 2, we present our moment-based shape descriptor and its three possible levels of intrinsic geometrical invariance, namely translation and scaling, similarity and affine transformations. In Sec. 3, we give the expression of the proposed multi-reference shape prior and present the evolution equations associated to each level of invariance. In Sec. 4, we illustrate the benefits of the new prior on the segmentation of objects with various topologies, undergoing large affine transformations, in the presence of noise, occlusions and clutter.

A preliminary version of this work was presented at the ECCV'06 conference [24].

\section{Shape Descriptors and Geometrical Invariance}

In our approach, objects are represented by their silhouette. In other words, shapes are defined by their closed boundaries or, equivalently, by their characteristic function, which is binary. This description is not very different from the signed distance function representation (see e.g. $[6,9]$ ) to 
which it is directly related. Both representations share an important property: they do not constrain shape topology. In other words, they can handle shapes made up of multiple connected components or shapes with holes. Our representation is also not bound to any particular implementation. Level sets [23] as well as spline snakes [20] implementations may thus be used. The originality of our approach with respect to shape representation is that we encode the characteristic function using moments. This provides a compact, parametric representation that can be made intrinsically invariant to affine transformations and from which a shape prior can be naturally defined in terms of distance or probability, as we will see in Sec. 3 .

\subsection{Encoding Shapes with Moments}

Denoting by $\Omega_{i n}$ the inside region of a shape, the regular or geometric moments of its characteristic function $f$ are defined as:

$$
M_{p, q}=\iint f(x, y) x^{p} y^{q} d x d y=\iint_{\Omega_{i n}} x^{p} y^{q} d x d y,
$$

where $(p, q) \in \mathbb{N}^{2}$, and $(p+q)$ is called the order of the moment. Any shape, discretized on a sufficiently fine grid, may be reconstructed from its infinite set of moments. Hence, when computed from the characteristic function, moments naturally provide region-based shape descriptors. However, as is well-known [25], a more tractable representation for reconstruction purposes is obtained by using an orthogonal basis, such as Legendre polynomials:

$$
\lambda_{p, q}=C_{p q} \iint_{\Omega_{i n}} P_{p}(x) P_{q}(y) d x d y,
$$

for $(x, y) \in[-1,1]^{2}$, where $C_{p q}=(2 p+1)(2 q+1) / 4$ is a normalizing constant, and for $x \in[-1,1]$ :

$$
P_{p}(x)=\sum_{k=0}^{p} a_{p k} x^{k}=\frac{1}{2^{p} p !} \frac{d^{p}}{d x^{p}}\left(x^{2}-1\right)^{p} .
$$

In practice we limit this representation to a finite order $N$. We define the shape descriptor as the $D$ dimensional vector of Legendre moments, $\left\{\lambda_{p, q}, p+q \leq N\right\}$, where $D=(N+1)(N+2) / 2$. Orthogonal moments rather than regular moments have to be considered since reconstructing a shape from a finite number of non orthogonal, geometric moments, involves inverting an ill-conditioned Gram matrix [26]: it amounts to recovering a vector from its components on a set of nearly parallel vectors. On the contrary, reconstruction from orthogonal moments does not suffer from ill-conditioning and is given by a direct, closed-form expression:

$$
\hat{f}_{N}(x, y)=\sum_{p=0}^{N} \sum_{q=0}^{p} \lambda_{p-q, q} P_{p-q}(x) P_{q}(y) .
$$

Let us also notice that, since the number of moments gathered in the descriptor is finite, the reconstruction of the characteristic function is not exact. As a consequence, the representation is not strictly reversible, but this is not required in the proposed method, because optimization is performed by curve evolution (see Sec. 3). Reconstructions using eq. (4) may be useful for choosing the order of the model (see Fig. 1) or for illustrating the accuracy of the representation (Fig. 2). 
Beyond their good numerical stability, orthogonal moments have another appealing property. When approximating a function using eq. (4) at the order $N$, the optimal coefficients for the reconstruction, in terms of Minimal Mean Square Error, are the Legendre moments given by eq. (2). As a consequence, Legendre moments define a hierarchical description of shape (see Fig. 1), whose accuracy increases with $N$. This can be exploited to ease the optimization procedure: a rough representation can be used in a first step and progressively refined by adding moments to the descriptor in a coarse-to-fine optimization scheme.

Finally, note that there is a linear relationship between Legendre moments and regular moments:

$$
\lambda_{p, q}=C_{p q} \sum_{u=0}^{p} \sum_{v=0}^{q} a_{p u} a_{q v} M_{u, v} .
$$

Computing Legendre moments using eq. (5) is preferable to eq. (2) because replacing the $M_{u, v}$ 's by properly chosen expressions yields geometrically invariant Legendre descriptors, as explained in the next subsection.

\subsection{Handling Pose and Geometric Variability}

A basic problem when using deformable shape models in image segmentation is shape alignment. The shape pose with respect to the underlying image has to be determined. In 2D, this involves transformations such as translation, scaling and rotation. The standard approach for shape alignment is to introduce explicit pose parameters in the optimization, which complicates the segmentation procedure. An alternative is to define intrinsically invariant shape descriptors. For this purpose, we defined in [21] the so-called canonical representation by a change of variables in which two shapes, differing by a given transformation, are represented by the same descriptor. This makes the model invariant w.r.t. the transformation in question. Since affine transformations include translation, scaling and rotation, this solves the alignment problem. Moreover, since transformations such as skewing and reflection are also included, this introduces geometrical variabilities in the model. The change of variables is given by closed-form expressions involving only geometric moments, i.e. the data at hand, so no additional optimization over pose parameters is necessary. Note that the alternate method of intrinsic alignment proposed in [16] has the same property.

Scale and Translation Invariance In the case of scaling and translation, the canonical representation of a shape is obtained by aligning its centroid, $(\bar{x}, \bar{y})$, with the center of the domain and normalizing its area, $\left|\Omega_{i n}\right|$, to a constant, $1 / \beta$. This amounts to using the normalized central moments

$\eta_{u, v}$ instead of the $M_{u, v}$ 's in (5), as proposed in [17]. The shape descriptor invariant to translation and isotropic scaling, $\boldsymbol{\lambda}=\left\{\lambda_{p, q}, p+q \leq N\right\}$, will be defined by:

$$
\lambda_{p, q}=C_{p q} \sum_{u=0}^{p} \sum_{v=0}^{q} a_{p u} a_{q v} \eta_{u, v}
$$

with:

$$
\eta_{u, v}=\iint_{\Omega_{i n}} \frac{(x-\bar{x})^{u}(y-\bar{y})^{v}}{\left(\beta\left|\Omega_{i n}\right|\right)^{(u+v+2) / 2}} d x d y
$$



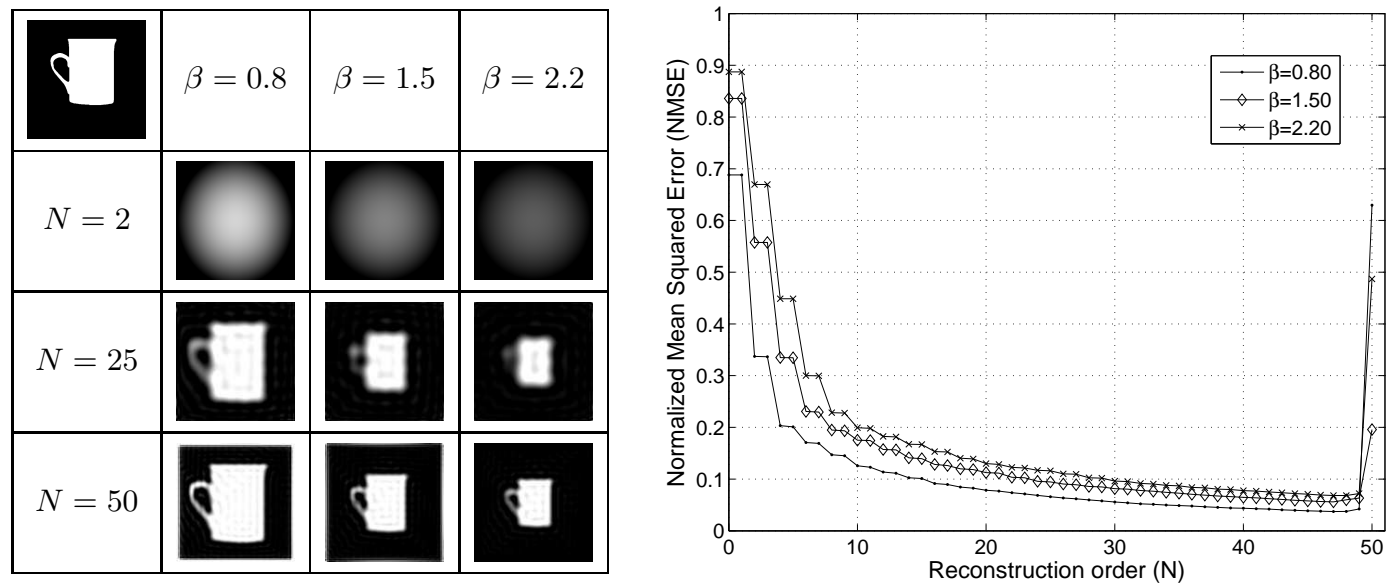

Fig. 1. Reconstructions of a mug shape from its Legendre moments made invariant w.r.t. scale and translation (left) and corresponding reconstruction error curves (right).

and

$$
\text { with } \quad \bar{x}=\frac{M_{1,0}}{M_{0,0}}, \bar{y}=\frac{M_{0,1}}{M_{0,0}} \text { and }\left|\Omega_{i n}\right|=M_{0,0} .
$$

Fig. 1 illustrates the reconstruction of a shape from its normalized central Legendre moments. On the left, we can notice that every reconstructed shape is centered in the reconstruction domain and that for a fixed $\beta$, all shapes have the same area. On the right, curves representing the Normalized Mean Square reconstruction Error (9) are presented.

$$
\operatorname{NMSE}(N)=\frac{\iint\left(\widehat{f}_{N}(x, y)-f(x, y)\right)^{2} d x d y}{\iint f(x, y)^{2} d x d y} .
$$

The sources of errors in the numerical evaluation of image moments are analyzed in [27,28]. As illustrated by Fig. 1 right, two of them are dominant in our case. The first one is the truncation error: the higher the order $N$, the better the reconstruction. The second one is related to numerical accuracy: for large $N$, numerical approximation errors prevail. For all our experiences we have used double-precision Matlab ${ }^{\circledR}$ implementations, representing numbers with 15 precision digits. Beyond order 48 , some coefficients of Legendre polynomials have more than 15 digits, which causes a sudden increase in the reconstruction error (see Fig. 1 right). We can also notice that reconstruction is better when $\beta$ is small. Choosing a small value for $\beta$ increases the area of the canonical representation, thus smaller details can be described with less moments. However, the canonical representation has to fit in the definition domain of Legendre moments, i.e. $[-1,1]^{2}$. As a consequence, for a given shape, there is a lower bound for $\beta$. To our knowledge, there are no theoretical results to calculate this bound. The value of $\beta$ is not critical, however. We have determined it experimentally for each shape. More examples are available in [29].

Affine Invariance In the case of affine invariance, the canonical representation is given by the image normalization procedure [30]. The original shape and its normalized version are related by an 

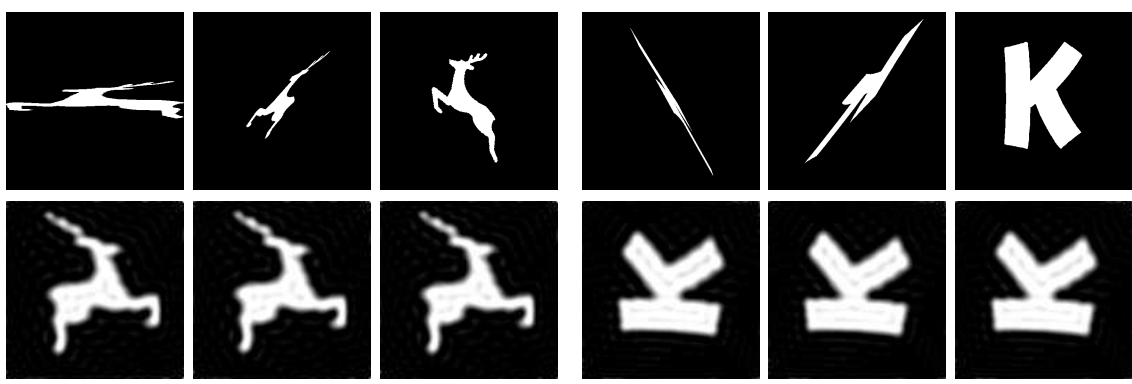

Fig. 2. Reconstruction of shapes from their affine-invariant moments. Upper row: original shapes. Lower row: shape reconstructions using affine invariant moments (these reconstruction correspond to the canonical representations of the original shapes).

affine transformation, i.e. a translation followed by a linear transformation which can be decomposed as:

$$
\left[\begin{array}{cc}
\cos \gamma & \sin \gamma \\
-\sin \gamma & \cos \gamma
\end{array}\right] \cdot\left[\begin{array}{ll}
l_{1} & 0 \\
0 & l_{2}
\end{array}\right] \cdot\left[\begin{array}{cc}
\cos \theta & \sin \theta \\
-\sin \theta & \cos \theta
\end{array}\right] .
$$

As already mentioned, the parameters of the change of variables $\left(\gamma, l_{1}, l 2, \theta, \bar{x}\right.$ and $\left.\bar{y}\right)$ are given by closed-form expressions involving geometric moments (these can be found in [21]). Image normalization, however, does not handle reflection. Since reflection only affects the sign of moments for $p$ odd (reflection w.r.t. $y$ axis) or for $q$ odd (reflection w.r.t. $x$ axis), we choose, without loss of generality, to fix the sign of the third-order moments. Finally, the affine-invariant descriptor, that will be denoted by $\boldsymbol{\lambda}^{A}$, is defined as [21]:

$$
\lambda_{p, q}^{A}\left(\Omega_{i n}\right)=C_{p q} \sum_{u, v}^{u \leq p, v \leq q} a_{p u} a_{q v} \eta_{u, v}^{A},
$$

where the affine-invariant moments are given by:

$$
\eta_{u, v}^{A}=\left(\operatorname{sign}\left(\widehat{\eta}_{3,0}^{A}\right)\right)^{u} \cdot\left(\operatorname{sign}\left(\widehat{\eta}_{0,3}^{A}\right)\right)^{v} \cdot \widehat{\eta}_{u, v}^{A}
$$

and:

$$
\begin{aligned}
& \widehat{\eta}_{u, v}^{A}=\frac{\left(l_{1} \cdot l_{2}\right)^{\frac{u+v}{4}}}{\left(\beta\left|\Omega_{i n}\right|\right)^{(u+v+2) / 2}} \\
& \times \iint_{\Omega_{i n}}\left(\frac{((x-\bar{x}) \cos \theta+(y-\bar{y}) \sin \theta)}{\sqrt{l_{1}}} \cos \gamma+\frac{((y-\bar{y}) \cos \theta-(x-\bar{x}) \sin \theta)}{\sqrt{l_{2}}} \sin \gamma\right)^{u} \\
& \times\left(\frac{((y-\bar{y}) \cos \theta-(x-\bar{x}) \sin \theta)}{\sqrt{l_{2}}} \cos \gamma-\frac{((x-\bar{x}) \cos \theta+(y-\bar{y}) \sin \theta)}{\sqrt{l_{1}}} \sin \gamma\right)^{v} d x d y .
\end{aligned}
$$

To illustrate the invariance of the proposed descriptor, we show on Fig. (2) examples of shape reconstruction with affine invariant descriptors. The initial shapes $(512 \times 512$ images $)$ shown on the upper row are affine-transformed versions of the "stag" and "K" shapes. While large affine deformations are considered, the reconstructions $(N=47,128 \times 128$ images $)$, are similar for each shape and correspond to their canonical representation. 
Similarity Invariance A simpler model that only handles similarities may readily be obtained by setting $\gamma=0$ and $l_{1}=l_{2}=1$ in (13):

$$
\lambda_{p, q}^{S}\left(\Omega_{i n}\right)=C_{p q} \sum_{u, v}^{u \leq p, v \leq q} a_{p u} a_{q v} \eta_{u, v}^{S}
$$

with:

$$
\eta_{u, v}^{S}=\left(\operatorname{sign}\left(\widehat{\eta}_{3,0}^{S}\right)\right)^{u} \cdot\left(\operatorname{sign}\left(\widehat{\eta}_{0,3}^{S}\right)\right)^{v} \cdot \widehat{\eta}_{u, v}^{S}
$$

and

$$
\widehat{\eta}_{u, v}^{S}=\iint_{\Omega_{i n}} \frac{((x-\bar{x}) \cos \theta+(y-\bar{y}) \sin \theta)^{u} \cdot((y-\bar{y}) \cos \theta-(x-\bar{x}) \sin \theta)^{v}}{\left(\beta\left|\Omega_{i n}\right|\right)^{(u+v+2) / 2}} d x d y .
$$

\section{Multi-Reference Shape Priors}

In this section, we define a shape prior based on Legendre descriptors. This prior takes into account either a single reference shape or a set of reference shapes. Depending on the choice of the descriptor $\boldsymbol{\lambda}, \boldsymbol{\lambda}^{\boldsymbol{S}}$ or $\boldsymbol{\lambda}^{\boldsymbol{A}}$, the proposed constraint is able to handle several levels of geometric invariance, as described in Section 2. The derivation of the evolution equation corresponding to the minimization of the prior is detailed first in the case of the single-reference model and then in the multi-reference case.

\subsection{Definition of the prior}

Let us first consider the case where the active contour, $\Gamma$, is constrained to evolve toward a single reference shape. It is natural to define a shape constraint as a distance $d$ in terms of shape descriptors. Equivalently, in a probabilistic framework, we define a shape prior energy as:

$$
J_{\text {prior }}\left(\Omega_{\text {in }}\right)=-\log \left(\mathcal{P}\left(\boldsymbol{\lambda}\left(\Omega_{\text {in }}\right)\right)\right),
$$

where $\Omega_{i n}$ is the inside region of $\Gamma$, and:

$$
\mathcal{P}(\boldsymbol{\lambda}) \propto \exp \left(-d\left(\boldsymbol{\lambda}, \boldsymbol{\lambda}^{r e f}\right)\right)
$$

where $\boldsymbol{\lambda}^{\text {ref }}$ is the set of moments of the reference object. In the simplest case $d$ is a quadratic distance. Of course, more elaborate expressions can be used to model arbitrarily complex priors. In particular, when $N_{r e f}$ reference shapes are simultaneously considered, the above model is extended by defining $\mathcal{P}(\boldsymbol{\lambda})$ as a mixture of pdf's. When $d$ is quadratic and all shapes are equiprobable, this leads to a mixture-of-Gaussians:

$$
\mathcal{P}(\boldsymbol{\lambda})=\frac{1}{N_{\text {ref }}} \frac{1}{\sigma \sqrt{2 \pi}} \sum_{k=1}^{N_{\text {ref }}} \exp \left(-\frac{\left\|\boldsymbol{\lambda}-\boldsymbol{\lambda}_{(k)}^{\text {ref }}\right\|^{2}}{2 \sigma^{2}}\right) .
$$

In this paper, we will consider multiple-reference models involving different fixed shapes. Let us notice that eq. (19) is close to the classical Parzen density estimator, thus the model readily extends to the definition of statistical shape variabilities, in the spirit of [16]. 


\subsection{Active Contour Evolution Equation for the Single-Reference Model}

The evolution equation for the boundary of $\Omega_{i n}$ can be derived from the minimization of $J_{\text {prior }}$ using the shape derivative framework [22]. Let us first focus on the case where the shape constraint is a quadratic distance to a single reference shape, described by $\boldsymbol{\lambda}^{\text {ref }}$, i.e.:

$$
J_{\text {prior }}\left(\Omega_{\text {in }}(t)\right)=\sum_{p, q}^{p+q \leq N}\left(\lambda_{p, q}\left(\Omega_{i n}(t)\right)-\lambda_{p, q}^{r e f}\right)^{2} .
$$

Scale and Translation Invariance When the descriptor is invariant w.r.t. location and scale, i.e. when $\boldsymbol{\lambda}$ and $\boldsymbol{\lambda}^{r e f}$ are computed from normalized central moments, applying the strategy described in [22] to minimize $J_{\text {prior }}$ leads to the following flow (see [31] for details):

$$
\frac{\partial \Gamma}{\partial t}=\underbrace{\sum_{u, v}^{u+v \leq N} A_{u v}\left(H_{u v}\left(x, y, \Omega_{i n}\right)+\sum_{i=0}^{2} B_{u v i} . L_{i}(x, y)\right)}_{V_{\text {prior }}} \mathcal{N}
$$

where $\mathcal{N}$ is the inward unit normal vector of the evolving active contour $\Gamma$ and:

$$
\begin{aligned}
& A_{u v}=2 \sum_{p, q}^{p+q \leq N}\left(\lambda_{p, q}-\lambda_{p, q}^{r e f}\right) C_{p q} a_{p u} a_{q v}, \\
& H_{u v}\left(x, y, \Omega_{i n}\right)=\frac{(x-\bar{x})^{u}(y-\bar{y})^{v}}{\left(\beta\left|\Omega_{i n}\right|\right)^{(u+v+2) / 2}} \\
& B_{u v 0}=\frac{u \cdot \bar{x} \cdot \eta_{u-1, v}+v \cdot \bar{y} \cdot \eta_{u, v-1}}{\beta^{\frac{1}{2}}\left|\Omega_{i n}\right|^{\frac{3}{2}}}-\frac{(u+v+2) \cdot \eta_{u, v}}{2\left|\Omega_{i n}\right|}, \\
& B_{u v 1}=\frac{-u \cdot \eta_{u-1, v}}{\beta^{\frac{1}{2}}\left|\Omega_{i n}\right|^{\frac{3}{2}}}, \quad B_{u v 2}=\frac{-v \cdot \eta_{u, v-1}}{\beta^{\frac{1}{2}}\left|\Omega_{i n}\right|^{\frac{3}{2}}} \\
& L_{0}=1, \quad L_{1}=x, \quad L_{2}=y .
\end{aligned}
$$

Fig. 3 illustrates the ability of the proposed shape prior to constrain an evolving shape to resemble a reference one, and to allow free changes of topology while the warping proceeds.

Similarity Invariance When taking into account similarity transforms in the prior, i.e.using $\lambda^{S}$, we obtain (see [29] for details):

$$
\frac{\partial \Gamma}{\partial t}=\underbrace{\sum_{u, v}^{u+v \leq N} A_{u v}^{S} \cdot\left(\operatorname{sign}\left(\widehat{\eta}_{3,0}^{S}\right)\right)^{u} \cdot\left(\operatorname{sign}\left(\widehat{\eta}_{0,3}^{S}\right)\right)^{v} \cdot\left(H_{u v}^{S}+\sum_{i=0}^{9} \widehat{B}_{u v i}^{S} \cdot L_{i}\right)}_{V_{\text {prior }}} \mathcal{N},
$$

where the expressions of all necessary coefficients are given in [31]. 


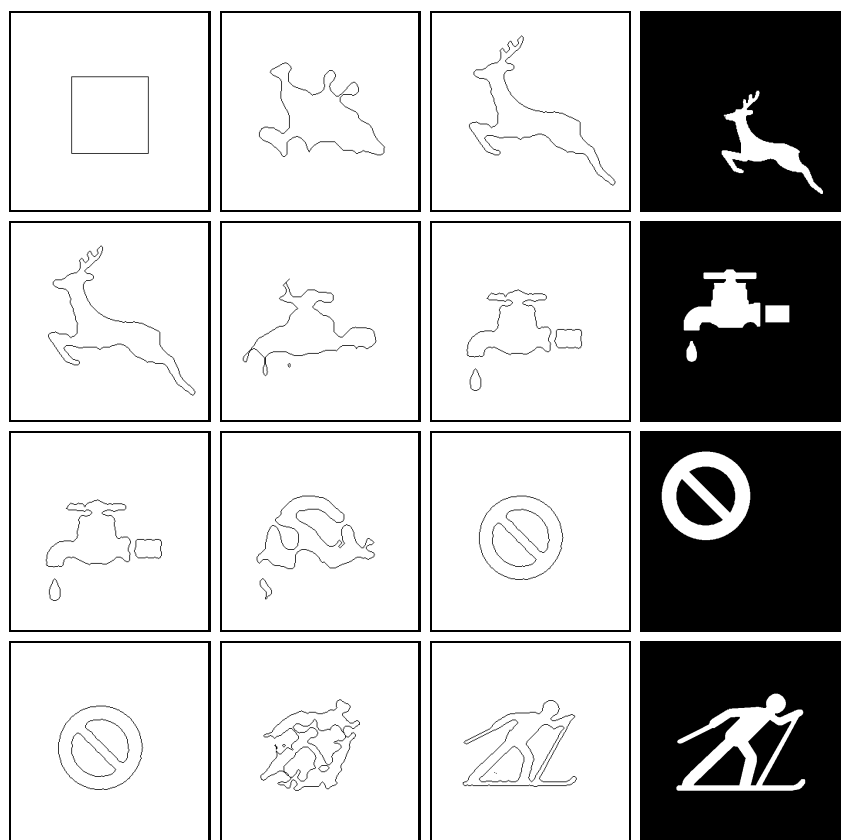

(a)

(b)

(c)

(d)

Fig. 3. Curve evolutions using the single-reference shape prior invariant with regard to translation and scaling: (a) initial curves, (b) intermediate evolutions, (c) final results and (d) reference shapes.

Affine Invariance When taking into account affine invariance in the prior, i.e.using $\boldsymbol{\lambda}^{\boldsymbol{A}}$, we obtain (see [29] for details):

$$
\frac{\partial \Gamma}{\partial t}=\underbrace{\sum_{u, v}^{u+v \leq N} A_{u v}^{A} \cdot\left(\operatorname{sign}\left(\widehat{\eta}_{3,0}^{A}\right)\right)^{u} \cdot\left(\operatorname{sign}\left(\widehat{\eta}_{0,3}^{A}\right)\right)^{v} \cdot\left(H_{u v}^{A}+\sum_{i=0}^{9} \widehat{B}_{u v i}^{A} \cdot L_{i}\right)}_{V_{\text {prior }}} \mathcal{N}
$$

where the expressions of all necessary coefficients are given in [31].

\subsection{Evolution equation for the Multi-Reference Model}

Let us now consider the multi-reference case. For the sake of conciseness, we present the case of translation and scale invariance, the cases of similarity and affine invariance being similar. Taking the $\log$ in eq. (19), the functional to be minimized is:

$$
J_{\text {prior }}^{\text {multi }}\left(\Omega_{\text {in }}(t)\right)=-\log \left[\sum_{k=1}^{N_{\text {ref }}} \exp \left(-\frac{\left\|\boldsymbol{\lambda}\left(\Omega_{\text {in }}\right)-\boldsymbol{\lambda}_{(k)}^{\text {ref }}\right\|^{2}}{2 \sigma^{2}}\right)\right]+c s t .
$$


Using elementary differentiation rules, we have (omitting $\Omega_{\text {in }}$ for conciseness):

$$
\frac{\partial J_{\text {prior }}^{\text {multi }}}{\partial t}=\frac{1}{2 \sigma^{2} \sum_{k=1}^{N_{\text {ref }}} \exp \left(-\frac{\left\|\boldsymbol{\lambda}-\boldsymbol{\lambda}_{(k)}^{\text {ref }}\right\|^{2}}{2 \sigma^{2}}\right)} \cdot \sum_{k=1}^{N_{\text {ref }}} \frac{\partial J_{\text {prior }}^{(k)}}{\partial t} \cdot \exp \left(-\frac{\left\|\boldsymbol{\lambda}-\boldsymbol{\lambda}_{(k)}^{\text {ref }}\right\|^{2}}{2 \sigma^{2}}\right)
$$

where

$$
J_{\text {prior }}^{(k)}=\left\|\boldsymbol{\lambda}-\boldsymbol{\lambda}_{(k)}^{r e f}\right\|^{2}
$$

corresponds to the quadratic prior in the single-reference case, for the $k$-th reference shape. Its derivative is given by:

$$
\frac{\partial J_{\text {prior }}^{(k)}}{\partial t}=\sum_{u, v}^{u+v \leq N} A_{(k) u v} . \delta_{E}\left(\eta_{u, v}\right)
$$

where

$$
A_{(k) u v}=2 \sum_{p, q}^{p+q \leq N}\left(\lambda_{p, q}-\lambda_{(k) p, q}^{r e f}\right) C_{p q} a_{p u} a_{q v} .
$$

Incorporating eq. (32) into (30) and exchanging summations, we obtain:

$$
\frac{\partial J_{\text {prior }}^{\text {multi }}}{\partial t}=\sum_{u, v}^{u+v \leq N} A_{u v}^{\text {multi }} \cdot \delta_{E}\left(\eta_{u, v}\right)
$$

with $A_{u v}^{\text {multi }}$ defined as in eq. (36). The rest of the derivation is similar to the single-reference case and we obtain an expression similar to (21), but with a different $A_{u, v}$ factor:

$$
\frac{\partial \Gamma}{\partial t}=\underbrace{\sum_{u, v}^{u+v \leq N} A_{u v}^{\text {multi }}\left(H_{u v}\left(x, y, \Omega_{i n}\right)+\sum_{i=0}^{2} B_{u v i} . L_{i}(x, y)\right)}_{V_{\text {prior }}} \mathcal{N}
$$

where the expressions of $H_{u v}, B_{u v i}$ and $L_{i}$ are given by equations (23) to (26). The $A_{u, v}^{\text {multi }}$ factor is a weighted average of the individual factors, $A_{(k) u v}$ computed for each reference shape descriptor $\lambda_{(k)}^{r e f}$ from $(22)$ :

$$
A_{u v}^{\text {multi }}=\frac{1}{2 \sigma^{2} \sum_{k=1}^{N_{\text {ref }}} \exp \left(\frac{-\left\|\boldsymbol{\lambda}-\boldsymbol{\lambda}_{(k)}^{r e f}\right\|^{2}}{2 \sigma^{2}}\right)} \sum_{k=1}^{N_{\text {ref }}} A_{(k) u v} \exp \left(\frac{-\left\|\boldsymbol{\lambda}-\boldsymbol{\lambda}_{(k)}^{r e f}\right\|^{2}}{2 \sigma^{2}}\right) .
$$

In other words, the force induced by the minimization of $J_{\text {prior }}$ in the multi-reference case is a weighted average of the individual forces directed toward each reference shape. Note that the weights decay exponentially with the distance in terms of shape descriptors between the evolving curve and the reference shape.

To summarize, the shape constraint we have proposed in this section handles complex topologies, simultaneously takes into account several reference shapes and is intrinsically invariant w.r.t affine transformations: the prior, as well the equation evolution, has a closed-form expression depending 
only on moments. Moreover, both the model and the derivation of the curve evolution equation are independent from any implementation consideration. Consequently, (21), (28) or (35) may be implemented using either a parametric approach, such as spline-snakes [20], or the non-parametric level sets formalism [23]. We use here the latter, which naturally handles changes of topology.

\section{Application to Image Segmentation}

To illustrate the behavior of the novel prior term in the general problem of two-class segmentation, we choose a standard data functional which was first introduced by Chan and Vese in [32]:

$$
J_{\text {data }}\left(\Omega_{\text {in }}, \Omega_{\text {out }}\right)=\iint_{\Omega_{\text {in }}}\left(I(x, y)-\mu_{\text {in }}\right)^{2} d x d y+\iint_{\Omega_{\text {out }}}\left(I(x, y)-\mu_{\text {out }}\right)^{2} d x d y,
$$

where $\mu_{\text {in }}$ (resp. $\mu_{\text {out }}$ ) is the (unknown) average intensity in the inside (resp. outside) domain, $\Omega_{\text {in }}$ (resp. $\Omega_{\text {out }}$ ), and $I(x, y)$ is the intensity value of the pixel. Its differentiation may be cast in the general framework presented in [22], which yields the following equation:

$$
\frac{\partial \Gamma(t)}{\partial t}=\underbrace{\left(I-\mu_{\text {in }}\right)^{2}-\left(I-\mu_{\text {out }}\right)^{2}}_{V_{\text {data }}}
$$

where $\mu_{\text {in }}$ and $\mu_{\text {out }}$ are updated after each iteration [32]. The global energy functional that we use for segmentation is then defined by:

$$
J\left(\Omega_{\text {in }}, \Omega_{\text {out }}\right)=\left(\frac{1}{1+\alpha}\right) J_{\text {data }}\left(\Omega_{\text {in }}, \Omega_{\text {out }}\right)+\left(\frac{\alpha}{1+\alpha}\right) J_{\text {prior }}\left(\Omega_{\text {in }}\right),
$$

which leads to equation (40) for the evolution of the contour.

$$
\frac{\partial \Gamma(t)}{\partial t}=\left[\left(\frac{1}{1+\alpha}\right) V_{d a t a}+\left(\frac{\alpha}{1+\alpha}\right) V_{\text {prior }}\right] \mathcal{N}
$$

where $V_{\text {prior }}$ is defined in $(21),(27),(28)$ or $(35)$, depending on the required level of invariance for the application and on the considered number of reference images. The geometric parameters are also updated after each iteration, using the appropriate expressions, e.g. eqs. (8) for invariance to scaling and translation, or the closed-form expressions given in [31] for invariance to similarity or affine transformations. Hence, pose parameters are obtained as a by-product of the method.

To illustrate how the model can take into account several reference shapes in a segmentation application, we first consider a reference set consisting of $N_{\text {ref }}=26$ letters, as shown in Fig. 4 . The parameter $\sigma$ is computed from the set $\left\{\boldsymbol{\lambda}_{(k)}^{r e f}\right\}$ in order to bound the probability of classification error, $\mathcal{P}_{e}$, between the two closest reference shapes in terms of descriptors, where:

$$
\mathcal{P}_{e}=\frac{1}{2} \operatorname{erfc}\left(\min _{k \neq l} \frac{\sqrt{\left\|\lambda_{(k)}^{r e f}-\lambda_{(l)}^{r e f}\right\|^{2}}}{2 \sigma \sqrt{2}}\right) .
$$

In practice, $\sigma$ is chosen so that $\mathcal{P}_{e}<3 \%$. 
We first consider six synthetic images (Fig. 5, first row). In these experiments, the model is invariant to scaling and translation i.e. we use $V_{\text {prior }}$ defined by eq. (35). The order $N$ of the model is chosen such that the Normalized Mean Squared reconstruction Error (NMSE) given by (9) is less than $10 \%$. The six segmentation results on the fourth row are obtained with the same curve evolution equation for the contour, i.e. the same value of $\alpha$ in eq. (40), with $V_{\text {prior }}$ given by eq. (35). Note that there is no need for any curvature term in this case: the multi-reference constraint is robust enough against the noise present in images. Also we can we can use (31) to identify each segmented letter. More results are given in Fig. 6, with the same set of reference shapes, the same level of geometric invariance and different images of synthetic letters featuring large occlusions.

\section{A

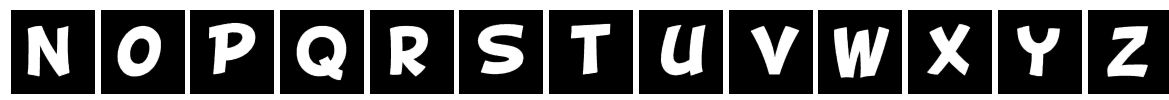

Fig. 4. Set of reference shapes used with the multi-reference model, eq. (19).

In Fig. 8, we present results on real data, using a different set of reference shapes: the twelve mugs shown on Fig. 7. Several kinds of initialization are used (first column). In the second column, we show the result of an evolution without shape prior, but with a standard additional curvature component. Then, starting from these results, we replace the curvature term by the shape prior up to the order 20 (third column). Last, we refine the results obtained at the order 20, using a model with moments up to the order 40 (fourth column). Note that when applying the shape prior, the same set of parameters ( $\alpha$ coefficient in eq. (40) and evolution step) is used for all experiments. On Fig. 8, we also give, for each segmentation stage, the computation time (including intermediary file savings and level sets-specific computations such as re-initialization of the host function) and the number of iterations using eq. (40), for this stage. All images have the same size: $474 \times 348$. The experiment was run under Matlab ${ }^{\circledR} 7.3$ on a laptop PC computer with an Intel ${ }^{\circledR}$ Core $2^{\mathrm{TM}}$ T7400 CPU at $2.16 \mathrm{GHz}$. Actually, a study of the complexity of the method, that was confirmed by measurements of computational times with various sets of parameters, shows that the computational burden is dominated by terms involving spatial coordinates such as the computation of regular moments or the computation of $V_{\text {prior }}$. The other computations, e.g. Legendre moments from regular moments, $A$ and $B$ coefficients, have a negligible impact on the overall complexity, which is $O\left(S^{2} N^{2}\right)$, where $S^{2}$ denotes the total number of pixels in the image and $N$ is the order of the model. Let us notice that using multiple references instead of a single reference shape [21] has little impact on the overall computation time since the only modification is the expression of the $A_{u, v}$ factors. For example, running the same experiment as on the third row of Fig. 8 , but with the single-reference model results in a global computation times of $285 \mathrm{~s}$ instead of 310 s for the multi-reference model (the number of iterations remains identical).

Fig. 9 shows a segmentation example using for the first time a multi-reference shape prior along with affine-invariance. The segmented image is the same as on the third line of Fig. 8 and the initialization is also identical. To show the ability of the multi-reference model to also handle affine invariance, we have modified the first reference shape (corresponding to the actual shape of Fig. 9a) 

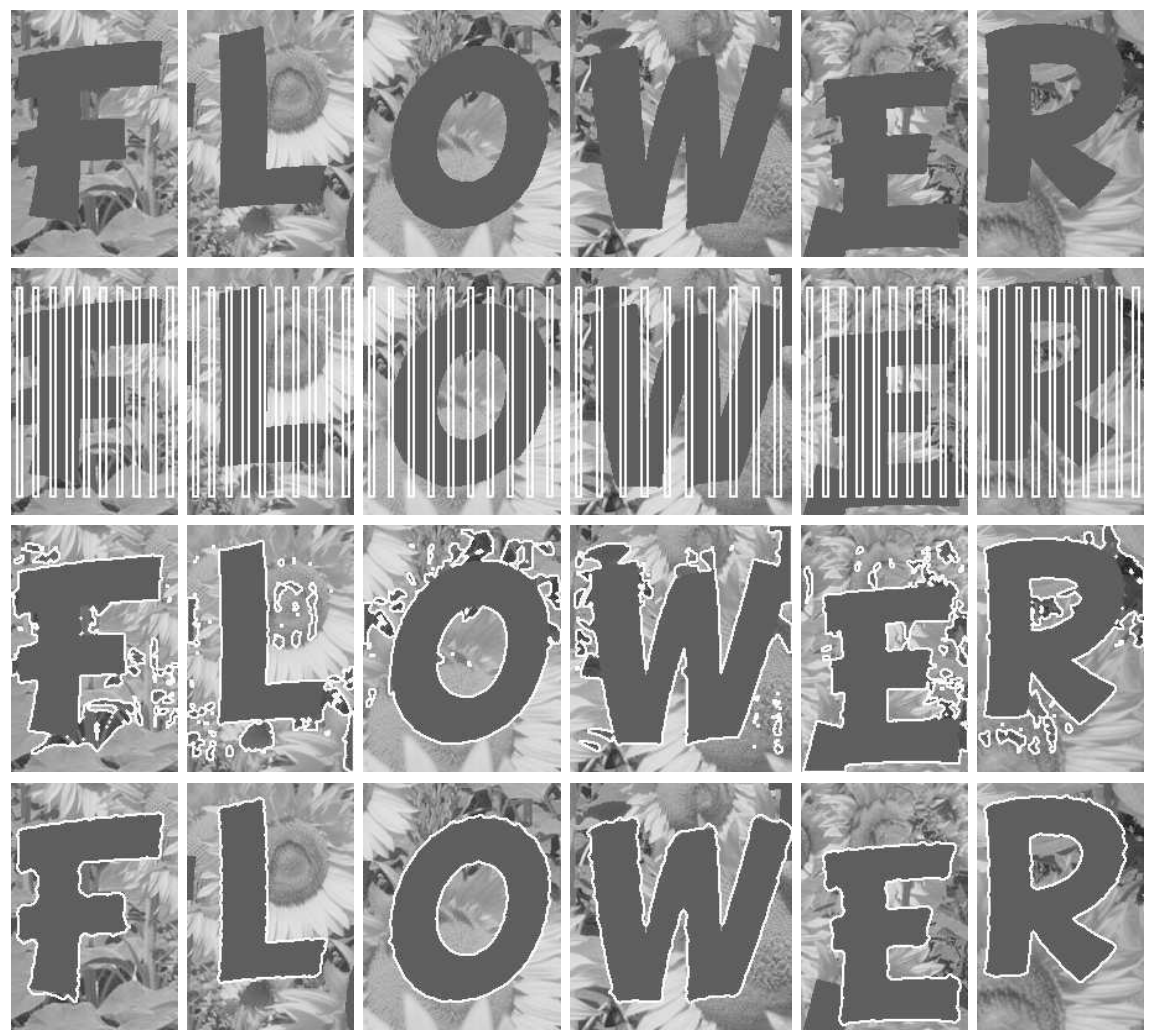

Fig. 5. Segmentation of synthetic images. First row: initial image, second row: initial contours, third row: segmentation results without shape prior, fourth row: segmentation result using the multi-reference prior invariant to scaling and translation: moments up to the 40th-order $(\beta=1.5)$.

by applying an anisotropic scaling to this reference (compare Fig. 9b to Fig.7). As can be observed (Fig. 9f), the final segmentation result is similar to the one in Fig. 8, though the exact shape is not present in the reference set in this case.

Fig. 10 illustrates the difficulty of the optimization problem, when combining both multiple references and affine invariance to constrain the evolution of a contour. For this most comprehensive model, the optimization problem naturally becomes intricate, due to the presence of many local minima in the objective function. Depending on the complexity of the observed image and of the reference shapes set, unexpected solutions may then occur, that often correspond to different relevant interpretations of the image to be segmented. On Fig. 10 the observed image corresponds to the letter "A" of the 26 letters alphabet, on which a partial occlusion has been superimposed. The first result, column (a), is obtained with the set of 26 reference shapes: the whole alphabet of Fig. 4. As can be seen, the model (and the optimization algorithm) favors in this case an evolution of the initial curve towards an inverted "V" rather than to the "desired" solution "A". Notice that even a human observer might interpret this silhouette as a deformed, bottom-up version of letter "V". Next, in 

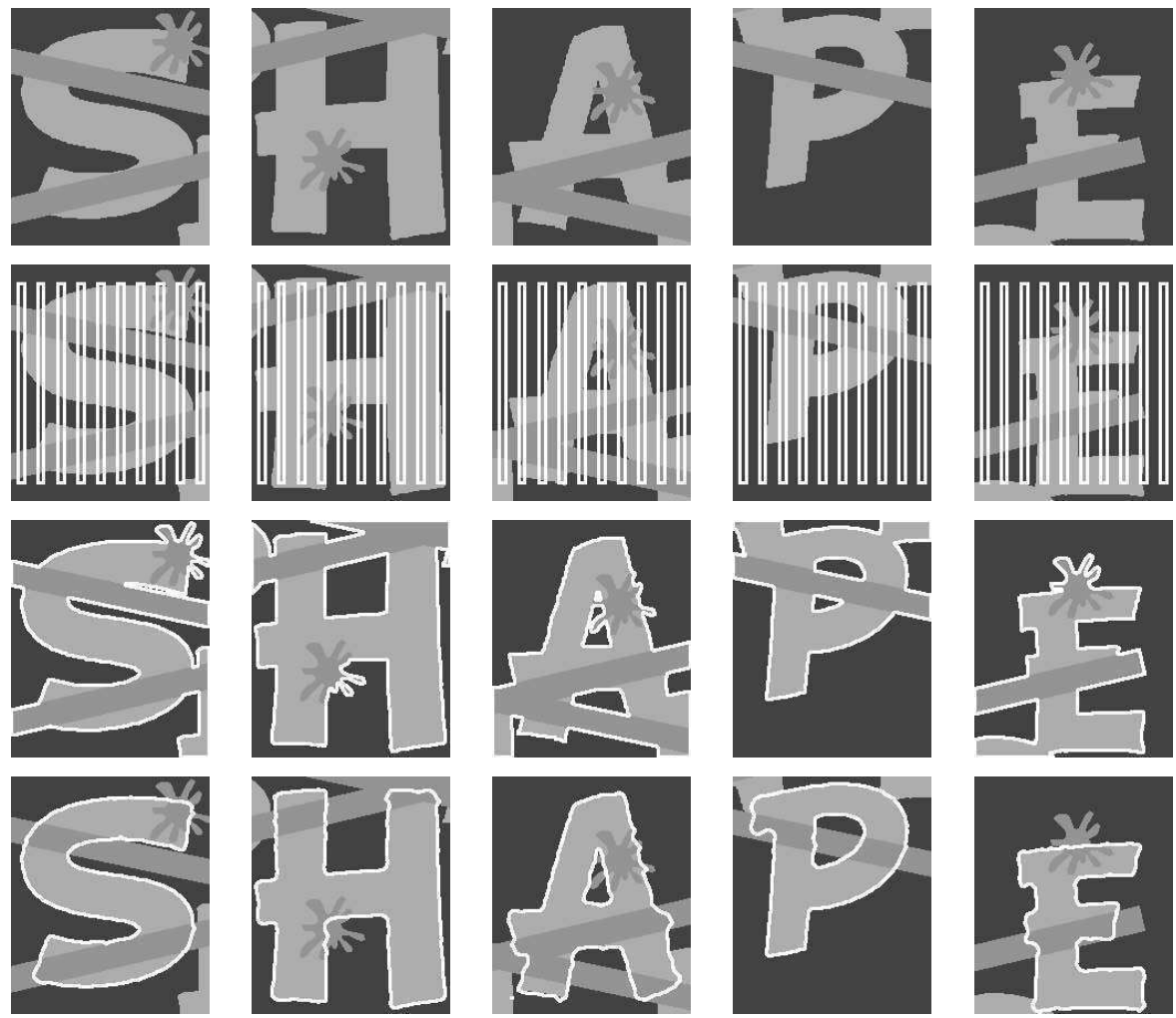

Fig. 6. Segmentation of five images of letters featuring large occlusions. First row: original images. Second row: initialization. Third row: results without shape constraint (no standard curvature component). Fourth row: final results, adding the multi-reference prior invariant to scaling and translation, up to the order 40 $(\beta=1.5)$. The same set of parameters is used for all the experiments.
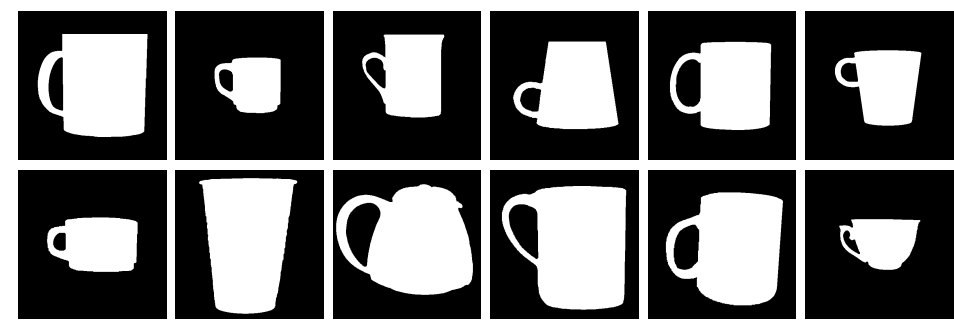

Fig. 7. Set of reference shapes used for the experience of Fig. 8.

column (b), the deformable model evolves using a representation that only takes into account scale and translation. This time, the final curve corresponds to a "A" letter, as expected, since the bottomup "V" letter does not belong to the solution space in this case. However, inspecting the final curve 
superimposed with the shape to be segmented, we notice that the two letters do not match perfectly because this simpler model does not handle rotation. In column (c), starting from the same initial curve, we evolve the contour using the affine-invariant model again, but this time the letter "V" is simply excluded from the reference alphabet (containing 25 letters in this case). As expected, the final curve correspond to the "desired" solution and matches accurately the original shape. Results presented columns (d) and (e) are obtained with the same experimental setting as in column (c), showing the ability of the model to handle large affine deformations and, in particular, reflections.

A last example (Fig. 11) shows the segmentation of a road sign on a color traffic image. The observation (Fig. 11b) is obtained from the RGB image (Fig. 11a) by computing the red coefficient

$r=\frac{R}{R+G+B}$ for every pixel. The road sign appears partially occluded and is rotated by approximately $27^{\circ}$ counterclockwise. The set of reference images includes rotated versions of the "stop" sign silhouette under 6 different angles: $-60^{\circ},-30^{\circ}, 0^{\circ}, 30^{\circ}, 60^{\circ}, 90^{\circ}$. Note that the exact observation angle is not present in the reference set. In this case, the invariance of the model has been limited to translation and scaling. As can be seen on Fig. 11g, the final segmentation is satisfactory and correspond to the desired solution. A similar experiment (which is not illustrated in this paper) was performed on this image with the same algorithm, using an extended reference set: one image every $2^{\circ}$ between $-88^{\circ}$ and $90^{\circ}$. The distance between every reference image and the final solution was computed according to (31). The closest reference image was the 58 -th one, which corresponds to a $26^{\circ}$ angle. These experiments show that it is possible to handle rotations through the definition of an appropriate set of reference images, while a lower level of geometric invariance is used, which naturally eases the optimization. This strategy is well suited to applications where the set of admissible geometric deformations is limited and known in advance.

\section{Conclusion}

In this paper, we have presented a novel approach for the integration of multiple prior shape models in active contour-based image segmentation. Our multi-reference prior shape model relies on affineinvariant shape descriptors related to Legendre moments. These descriptors are used to constrain the evolution of the contour towards a set of different possible reference shapes. A unique evolution equation for the active contour is derived, using the formalism of shape derivative. The corresponding geometric flow may be implemented with any contour evolution algorithm.

Experimental results obtained with the level sets implementation have shown that the proposed evolution equation introduces noticeable robustness to background clutter and occlusions in twoclass image segmentation problems. The proposed approach naturally handles pose variations, affine deformations and complex changes of topology. It is also suited to different kinds of initializations, which may be tailored to the application at hand.

As stated in Sec. 3, the mixture-of-Gaussian model that we use readily extends to Parzen density estimation. This paves the way for modeling statistical shape variabilities, which is an immediate perspective of this work.

Some issues remain open. A standard problem of active contour-based image segmentation is the choice of an adequate initialization, converging toward the "desired" solution. Another question is the determination of the model parameters. Two parameters had to be tuned manually here: the usual parameter $\alpha$ weighting the prior model and the data term, and the the step in the gradient descent. We noticed that the final solution was quite robust with respect to the choice of these parameters and the same parameters were used when segmenting different competing shapes in 
the same image using the multi-reference model. These parameters had however to be adapted from one image to the other. Another limitation of the proposed approach is that it only enables the segmentation of one single shape at the same time (even if multiple references are taken into account). An extension toward simultaneous (multiple) shapes segmentation is an intricate problem, which has been addressed recently by Cremers et al. [33] in the level sets framework, using multiple competing shape priors. Finally a 3D extension of this approach, in applications such as 3D computer vision or 3D medical imaging, would certainly stir large interest. Although such an extension seems quite straightforward from a mathematical point of view, difficulties may be expected in the optimization process, due to significant additional complexity in the energy landscape.

\section{References}

1. Terzopoulos, D., Metaxas, D.: Dynamic 3D models with local and global deformations: Deformable superquadrics. IEEE Transactions on Pattern Analysis and Machine Intelligence 13(7) (1991) 703-714

2. Staib, L., Duncan, J.: Boundary finding with parametrically deformable models. IEEE Transactions on Pattern Analysis and Machine Intelligence 14(11) (1992) 1061-1075

3. Cootes, T., Cooper, D., Taylor, C., Graham, J.: Active shape models - their training and application. Computer Vision and Image Understanding 61(1) (1995) 38-59

4. Székely, G., Kelemen, A., Brechbüler, C., Gerig, G.: Segmentation of 2D and 3D objects from MRI volume data using constrained elastic deformations of flexible Fourier surface models. Medical Image Analysis 1(1) (1996) 19-34

5. Cremers, D., Kohlberger, T., Schnörr, C.: Shape statistics in kernel space for variational image segmentation. Pattern Recognition : Special Issue on Kernel and Subspace Methods in Computer Vision 36(9) (2003) 1929-1943

6. Leventon, M., Grimson, W., Faugeras, O.: Statistical shape influence in geodesic active contours. In: Proc. of IEEE Conference on Computer Vision and Pattern Recognition, Hilton Head Island, Southern Carolina, USA (2000) 1316-1323

7. Rousson, M., Paragios, N.: Shape priors for level set representations. In: Proc. of 7th European Conference on Computer Vision, Lecture Notes in Computer Science. Volume 2351., Copenhaguen, Denmark (2002) 78-93

8. Riklin-Raviv, T., Kiryati, N., Sochen, N.: Unlevel-sets : geometry and prior-based segmentation. In: Proc. of the 8th European Conference on Computer Vision, Lecture Notes in Computer Science. Volume 3024., Prague, Czech Republic (2004) 50-61

9. Tsai, A., Yezzi, A., Wells, W., Tempany, C., Tucker, D., Fan, A., Grimson, W., Willsky, A.: A shapebased approach to the segmentation of medical imagery using level sets. IEEE Transactions on Medical Imaging 22(2) (2003) 137-154

10. Mansouri, A., Mukherjee, D., Acton, S.: Constraining active contour evolution via Lie groups of transformation. IEEE Transactions on Image Processing 13(6) (2004) 853-863

11. Unal, G., Krim, H., Yezzi, A.: Stochastic differential equations and geometric flows. IEEE Transactions on Image Processing 11(12) (2002) 1405-1416

12. Cremers, D., Tischhäuser, F., Weickert, J., Schnörr, C.: Diffusion snakes: Introducing statistical shape knowledge into the Mumford-Shah functional. International Journal of Computer Vision 50(3) (2002) 295-313

13. Chen, Y., Tagare, H., Thiruvenkadam, S., Huang, F., Wilson, D., Gopinath, K., Briggs, R., Geiser, E.: Using prior shapes in geometric active contours in a variational framework. International Journal of Computer Vision 50(3) (2002) 315-328

14. Bresson, X., Vandergheynst, P., Thiran, J.P.: A variational model for object segmentation using boundary information and shape prior driven by the mumford-shah functional. International Journal of Computer Vision 68(2) (2006) 145-162 
15. Zhang, T., Freedman, D.: Tracking objects using density matching and shape priors. In: Proc. of 9th IEEE International Conference on Computer Vision, Nice, France (2003) 1056-1062

16. Cremers, D., Osher, S.J., Soatto, S.: Kernel density estimation and intrinsic alignment for shape priors in level set segmentation. Int. J. Comput. Vision 69(3) (2006) 335-351

17. Foulonneau, A., Charbonnier, P., Heitz, F.: Geometric shape priors for region-based active contours. In: Proc. of IEEE Conference on Image Processing. Volume 3., Barcelona, Spain (2003) 413-416

18. Gastaud, M., Aubert, G., Barlaud, M.: Tracking video objects using active contours and geometric priors. Technical Report RR-2003-07-FR, I3S, Nice-Sophia Antipolis, France (2003)

19. Joshi, S., Mio, W., Srivastava, A., Liu, X.: Statistical shape analysis: Clustering, learning, and testing. IEEE Trans. Pattern Anal. Mach. Intell. 27(4) (2005) 590-602

20. Precioso, F., Barlaud, M.: B-spline active contour with handling of topology changes for fast video segmentation. Eurasip Journal on Applied Signal Processing, special issue: image analysis for multimedia interactive services - PART II 2002(6) (2002) 555-560

21. Foulonneau, A., Charbonnier, P., Heitz, F.: Affine-invariant geometric shape priors for region-based active contours. IEEE Transactions On Pattern Analysis and Machine Intelligence 28(8) (2006) 13521357

22. Aubert, G., Barlaud, M., Faugeras, O., Jehan-Besson, S.: Image segmentation using active contours: calculus of variations or shape gradients? SIAM Journal on Applied Mathematics 63(6) (2003) 2128-2154

23. Osher, S., Sethian, J.: Fronts propagating with curvature-dependent speed: algorithms based on Hamilton-Jacobi formulations. Journal of Computational Physics 79(1) (1988) 12-49

24. Foulonneau, A., Charbonnier, P., Heitz, F.: Affine-invariant multi-reference shape priors for active contours. In: Computer Vision - ECCV 2006. Volume 3952 of LNCS., Springer (2006) 601-613

25. Teague, M.: Image analysis via the general theory of moments. Journal of the Optical Society of America 70(8) (1980) 920-930

26. Talenti, G.: Recovering a function from a finite number of moments. Inverse Problems 3 (1987) 501-517

27. Liao, S., Pawlak, M.: On image analysis by moments. IEEE Transactions on Pattern Analysis and Machine Intelligence 18(3) (1996) 254-266

28. Mukundan, R., Ramakrishnan, K.: Moments functions in image analysis - Theory and applications. World Scientific Publishing Co, Pte Ltd, Singapore (1998) ISBN 981-02-3524-0.

29. Foulonneau, A.: A contribution to the introduction of geometric shape contraints in region-based active contours (in French). PhD thesis, University Louis Pasteur, Strasbourg I (2004) Available online: http://lsiit-miv.u-strasbg.fr/lsiit/perso/Charbonnier.htm.

30. Pei, S., Lin, C.: Image normalization for pattern recognition. Image and Vision Computing 13(10) (1995) 711-723

31. Foulonneau, A., Charbonnier, P., Heitz, F.: Multi-reference affine-invariant geometric shape priors for region-based active contours. Technical Report RR-AF01-08, LRPC ERA 27 LCPC / LSIIT UMR 7005 CNRS (2008) Available online: http://lsiit.u-strasbg.fr/Publications/2008/FCH08/RR-AF01-08.pdf.

32. Chan, T., Vese, L.: Active contours without edges. IEEE Transactions on Image Processing 10(2) (2001) 266-277

33. Cremers, D., Sochen, N., Schnörr, C.: A multiphase dynamic labeling model for variational recognitiondriven image segmentation. Int. J. Comput. Vision 66(1) (2006) 67-81 

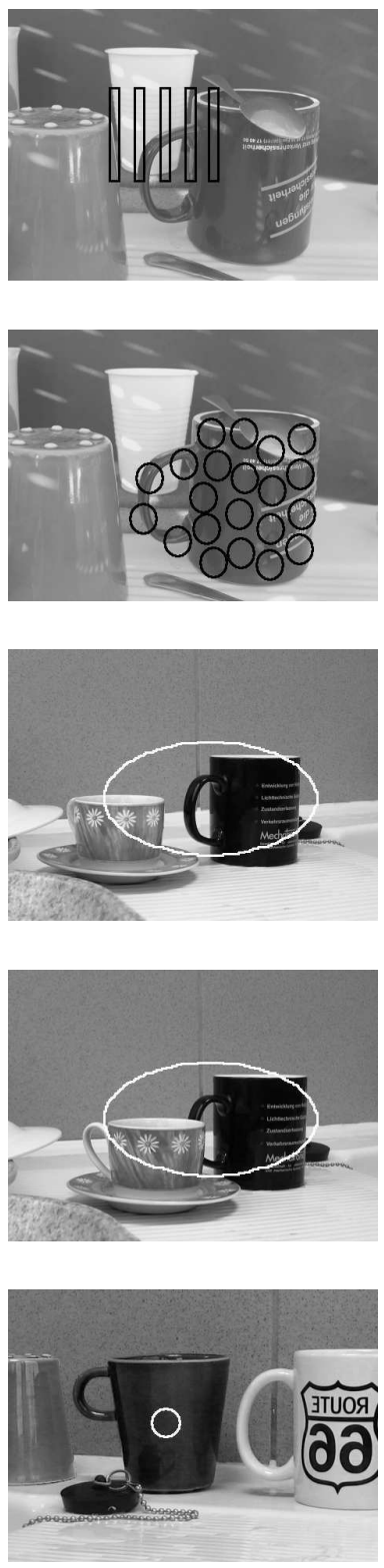

(a)

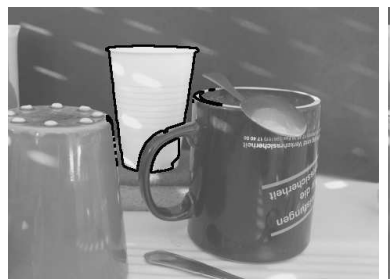

39 iterations, $21 \mathrm{~s}$

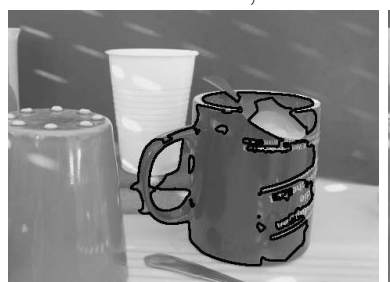

90 iterations, 65 s

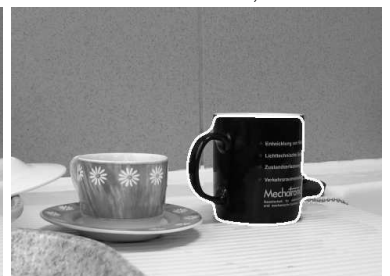

106 iterations, 56s

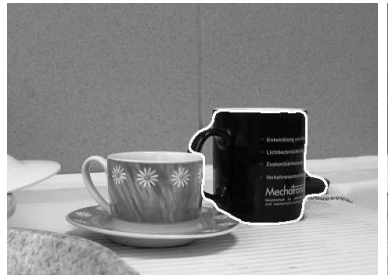

182 iterations, $97 \mathrm{~s}$

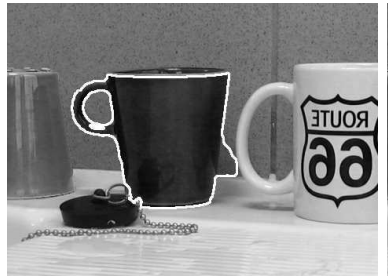

110 iterations, $58 \mathrm{~s}$

(b)

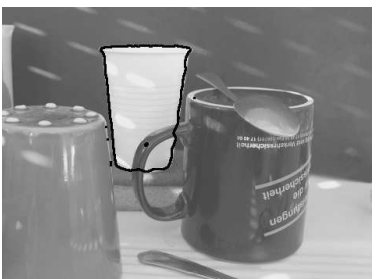

40 iterations, $52 \mathrm{~s}$

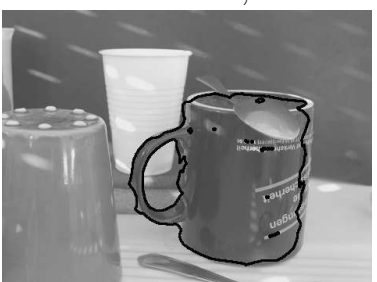

50 iterations, $122 \mathrm{~s}$

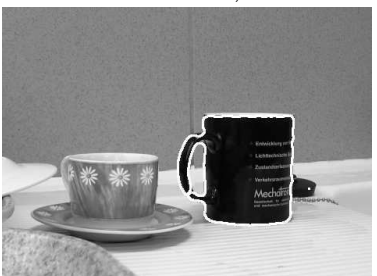

108 iterations, 134s

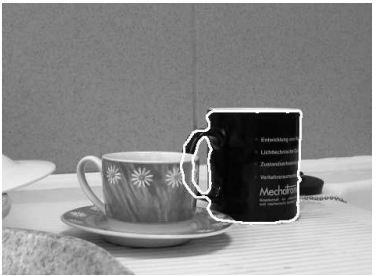

85 iterations, 109s

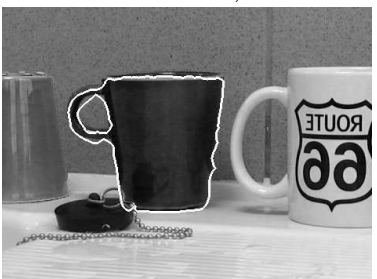

39 iterations, 59s

(c)

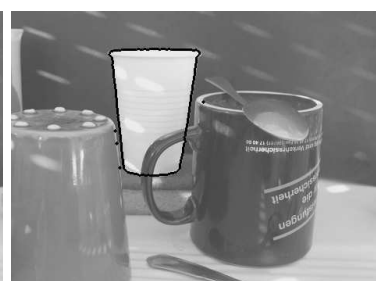

8 iterations, 27s

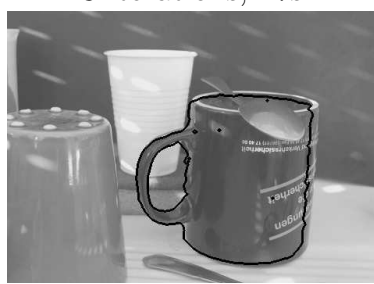

17 iterations, $97 \mathrm{~s}$

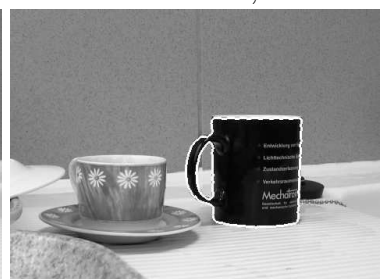

30 iterations, 120 s

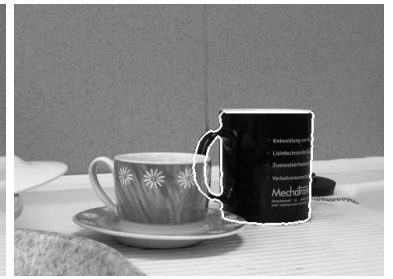

16 iterations, $64 \mathrm{~s}$

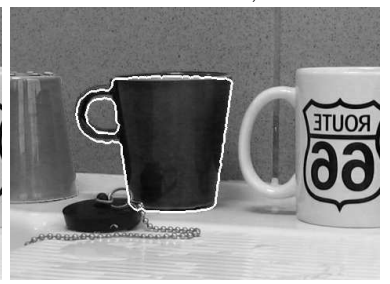

13 iterations, 58s

(d)

Fig. 8. Segmentation of real images. (a) Initial contours, (b) segmentation results without shape prior (standard curvature component used), segmentation result using the multi-reference prior invariant to scaling and translation: (c) moments up to the 20th-order and (d) to the 40th-order $(\beta=0.8)$. The number of evolution iterations performed at each stage and the corresponding computation time are given underneath each result. 


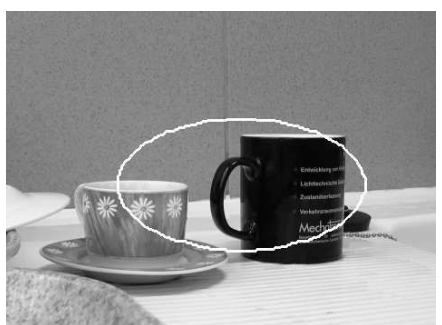

(a)

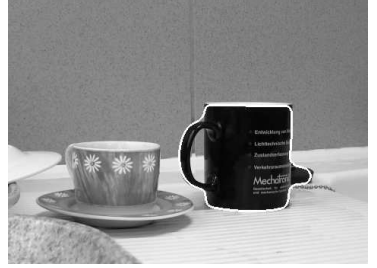

(c)
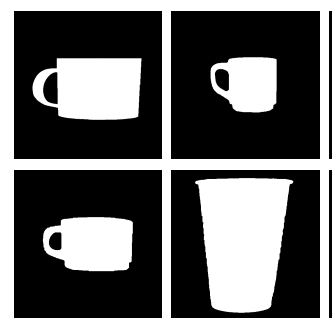

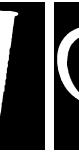

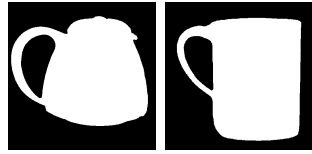

(b)

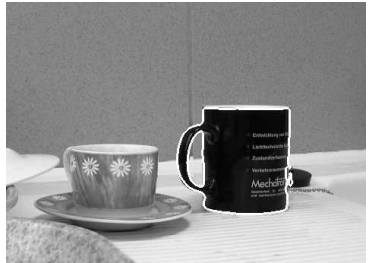

(d)

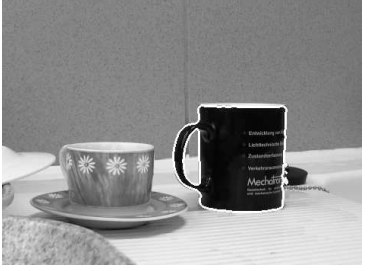

(e)
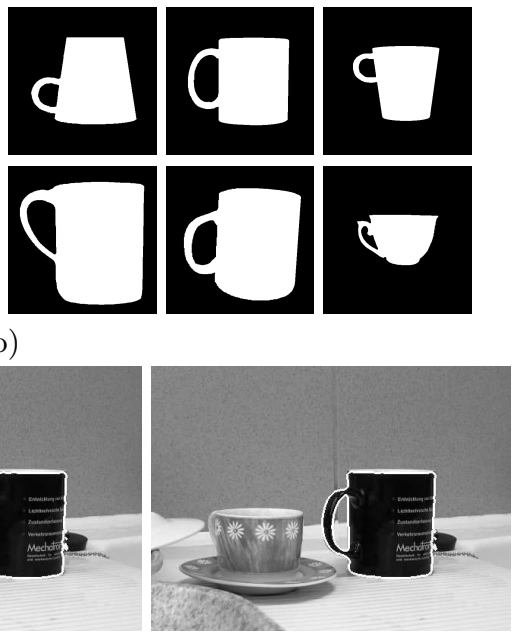

(f)

Fig. 9. Segmentation of a real image. (a) Initial curve ; (b) set of reference images ; (c) segmentation without shape constraint (standard curvature component used) ; segmentation result using the multi-reference, affineinvariant shape prior: (d) moments up to the 18th order, (e) to the 30 th order and (f) to the 40th order. 

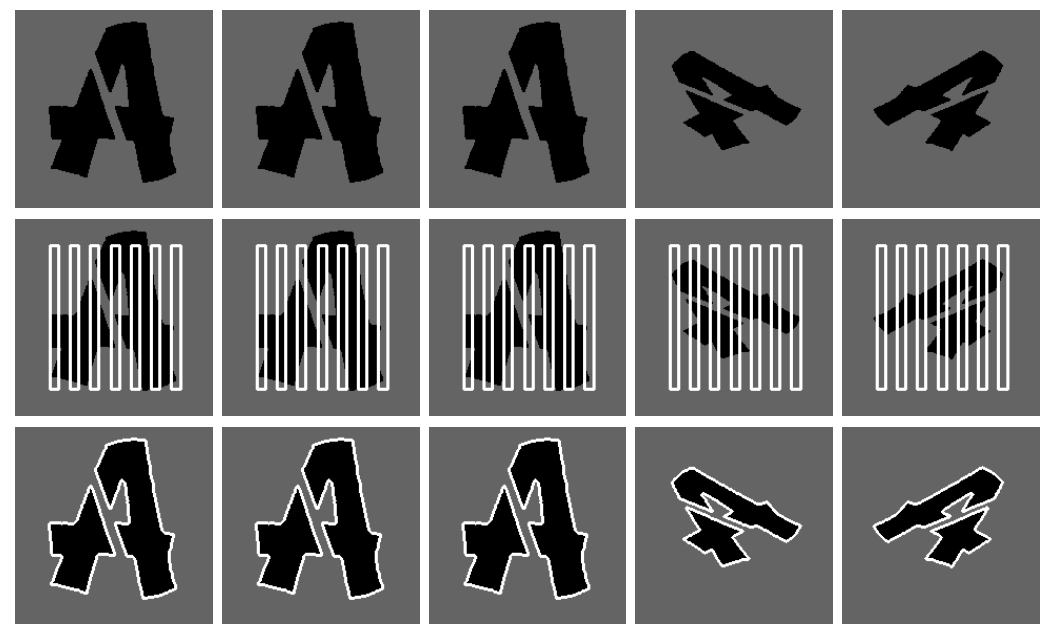

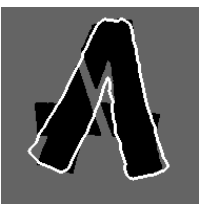

(a)

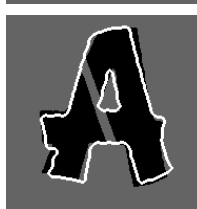

(b)

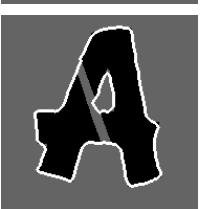

(c)

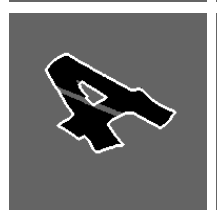

(d)

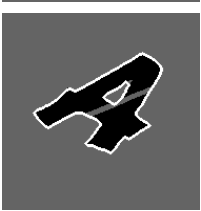

(e)

Fig. 10. Segmentation of synthetic images with the multi-reference prior using different levels of invariance and different set of reference shapes. First line: original images ; second line: initial curves ; third line: segmentation results without any prior ; fourth line: final segmentation results $(N=45)$. (a) the multireference shape prior is affine-invariant and simultaneously takes into account the 26 letters of the alphabet presented in Fig. 4. (b) the same set of reference shapes is used but invariance is limited to translation and scale. (c), (d), (e) the multi-reference shape prior is affine-invariant but the set of reference shapes does not include the "V" letter. 


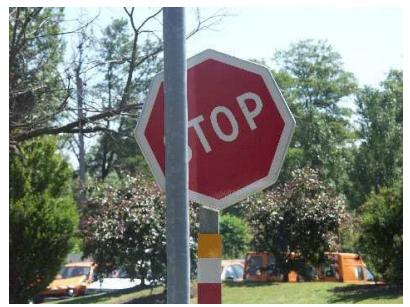

(a)

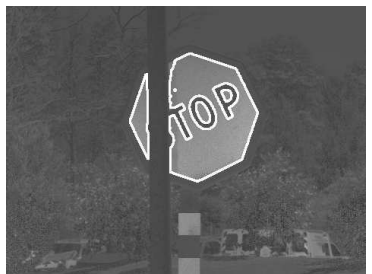

(d)

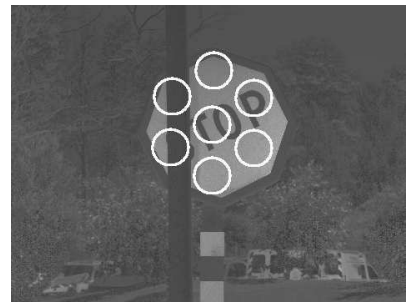

(b)
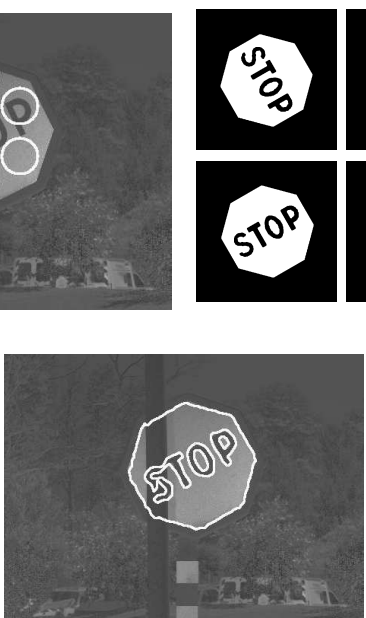

(f)

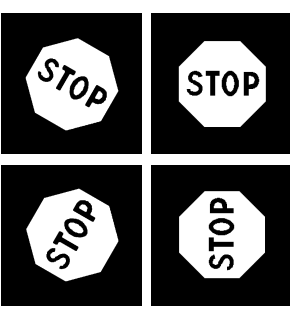

(c)

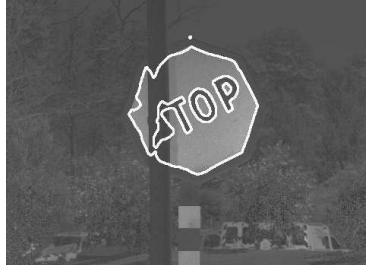

(e)

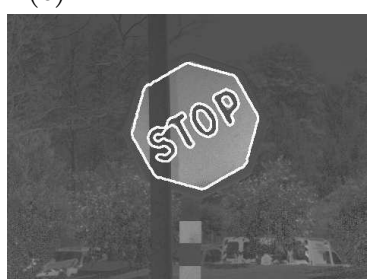

(g)

Fig. 11. First row: (a) original RGB image ; (b) corresponding normalized red component, $r=\frac{R}{R+G+B}$, and initial curve ; (c) reference images. Second row: (d) segmentation result without shape prior (no curvature component) ; (e)-(g) refinement of the segmentation using the multi-reference shape prior invariant to translation and scaling $(\beta=0.5)$. A progressively increasing order of representation is used : (e) $N=10,(\mathrm{f})$ $N=30$ and $(\mathrm{g}) N=42$. 